\title{
A Comparison of Paraxial and Extended Paraxial Approach in Laser Propagation and Second Harmonic Generation
}

\author{
P. SHARMA* \\ Ujjain Engineering College, Ujjain (M.P.) - 465010, India \\ (Received December 7, 2011; in final form April 25, 2012)
}

\begin{abstract}
The propagation of laser beam in the plasma has been investigated for two cases, first with imposing the restriction of paraxial approximation on the beam profile, and second, relaxing the restriction on the profile up to a certain extent. The beam width parameter of the propagating laser beam has been compared for both the cases. Since the off-paraxial part of the beam affects background densities, consequently it will also influence the 2nd harmonic generation. Therefore, comparison of results has been done on the generated second harmonic, in the light of these two cases. A notable change is found in the magnitude of second harmonic yield in modified-paraxial case as compared to the paraxial case.
\end{abstract}

PACS: 52.38.-r, 52.38.Hb, 52.38.Mf

\section{Introduction}

To explore maximum energy from the laser beam (in all laser-plasma applications), it is vital to understand and be able to control the nonlinear processes that affect the laser beams as they propagate through the plasma. The high power laser beam is capable of introducing various types of nonlinearities (thermal, ponderomotive and relativistic) during its propagation in the plasma and creates a refractive index profile in the plasma across its intensity profile. As a result, the laser beam perceives a lens-like medium and focuses by its own influence on itself and undergoes self-focusing. A laser beam with random fluctuations of intensity over its profile can also focus into the lens-like regions and the resulting phenomenon is known as the filamentation [1] of the beam. This phenomenon can lead to break-up of the incident beam into intense filaments. Conventionally, solutions for the laser beams can easily be obtained in the paraxial approximation of the wave equation and it is assumed that the paraxial wave equation gives an accurate description for wave beams near the axis throughout the propagation.

It is important to mention here that once the laser beam gets filamented, the paraxial approximation breaks down in filament where the focusing intensity is above a certain level. Hence in this situation paraxial approximation is less accurate and some corrections are necessary. The theory given by Alkhamanov et al. [2] have demonstrated that in the limit when eikonal is expanded only up to second power of $r$, the shape of the radial intensity profile remains unchanged. This approximation is inadequate when the radial electron density varies sharply with $r$ in the near axis region and slowly afterwards.

However, in some experimental situation results in laser plasmas are still not understood. The comparison

* e-mail: preranaiitd@rediffmail.com with experiments performed does not show reasonable agreement with the theoretical predictions.

Some experimental situation needs to go beyond the paraxial approximation such as some solid-state lasers or semiconductor injection lasers with a thin active layer, generate wide-angle beams for which the predictions of the paraxial approximation are often not sufficiently accurate. Moreover, the use of diffractive optical elements that present small features or of tightly focusing laser beams to reach the nonlinear range of intensities may lead to optical fields that cannot be accurately described within the paraxial approximation.

In addition to that, recent advances in laser technology, based on the chirped pulse amplification technique, make intensities greater than $10^{18} \mathrm{~W} / \mathrm{cm}^{2}$ available for experiments. In these experiments, the laser beam power can be 1000 times larger than the critical value and one can expect multiple filamentation, focusing up to the point where the paraxial approximation breaks down in every filament and radiation scatters. Hence, the paraxial approximation is less accurate for describing many phenomena of laser plasma interaction in which the laser beam gets filamented.

Therefore the present work has been justified by using extended paraxial approximation. In theoretical studies, phenomenon of second and third harmonic generation has been studied by many groups. Sharma et al. [3] have studied second harmonic generation by considering both relativistic and ponderomotive nonlinearities but with paraxial approximation. Gupta et al. [4] have studied the third harmonic generation by considering relativistic and ponderomotive nonlinearities. Sharma et al. [5] have studied stochastic heating of electrons by considering only ponderomotive nonlinearity with non-paraxial approximation. Sharma et al. [6] have investigated the laser beam filamentation and stochastic electron heating at upper hybrid layer with both the relativistic and ponderomotive nonlinearity by considering paraxial approx- 
imation. But no attempt has been made to compare the paraxial and nonparaxial approach in the laser plasma interaction to study the phenomenon of filamentation and harmonic generation.

In the present paper, we examined the beam width parameter of the laser beam when both relativistic and ponderomotive nonlinearities are operative simultaneously. The work has been justified by modifying the paraxial profile of the beam up to a certain level. The higher order teams in the expansion of dielectric constant and eikonal have been taken into account. The laser intensity profile and other relevant quantities of plasma were expanded up to the 4th power of radial distance $r$ and it makes a significant difference in the study of propagation of laser beam in plasma.

To examine the effect of using higher order terms on the other nonlinear processes the present work has been extended to study second harmonic generation. It is observed that in extended paraxial case the filaments of the laser beam have been split [4] and these modified structures affect the plasma wave propagation [7] and consequently harmonic generation $[8,9]$. Therefore, the power of second-harmonic generation has been determined and compared for both paraxial and modified paraxial cases. It is observed that due to the presence of off-axis part of the laser beam second-harmonic yield is affected significantly.

The paper is organized as follows. In Sect. 2, the laser beam propagation with modified or extended paraxial approximation has been discussed and the expression for coupling between laser beam and electron plasma wave due to relativistic and ponderomotive nonlinearities is deduced. In Sect. 3, the second harmonic generation is discussed and the expression for second harmonic power is deduced by taking off axial rays into account. The second harmonic yield has been compared for both paraxial and extended paraxial case. Conclusion is presented in Sect. 4.

\section{Formulation: laser beam and plasma wave}

The wave equation governing the electric vector of the laser beam of frequency $\omega_{0}$ is given by

$$
\frac{\partial^{2} E}{\partial Z^{2}}+\frac{1}{r} \frac{\partial E}{\partial r}+\frac{\partial^{2} E}{\partial r^{2}}+\frac{\omega_{0}^{2}}{c^{2}} \varepsilon E=0 .
$$

The initial intensity distributions of the beam is assumed as

$$
E E^{*}=E_{00}^{2} \mathrm{e}^{-r^{2} / r_{0}^{2}},
$$

where $r$ is the radial coordinate of the cylindrical coordinate system and $r_{0}$ is the initial beam width, $E_{00}$ is the axial amplitude of the beam and $\varepsilon$ is the effective dielectric constant [3]. In order to solve the above equation, we can express $E$ as

$$
E=A_{0}(r, z) \exp \left(-\mathrm{i} S_{0}(r, z)\right),
$$

where $A_{0}$ and eikonal $S_{0}$ are real. On solving (1) using the standard technique, $A_{0}$ and $S_{0}$ can be assumed as $[2-5]$ :

$$
A_{0}^{2}=\left(1+\frac{\alpha_{00} r^{2}}{r_{0}^{2} f_{0}^{2}}+\frac{\alpha_{02} r^{4}}{r_{0}^{4} f_{0}^{4}}\right) \frac{E_{00}^{2}}{f_{0}^{2}} \mathrm{e}^{-r^{2} / r_{0}^{2} f_{0}^{2}}
$$

and eikonal $S_{0}[2,4]$ as

$$
S_{0}=\frac{S_{00}}{r_{0}^{2}}+\frac{S_{02} r^{4}}{r_{0}^{4}} \quad \text { with } \quad S_{00}=\frac{r^{2}}{2 f_{0}} \frac{\mathrm{d} f_{0}}{\mathrm{~d} z},
$$

where $\alpha_{00}, \alpha_{02}$ are the coefficients of $r^{2}$ and $r^{4}$, respectively, and $S_{00}, S_{02}$ are the slowly varying function of $r$ and $z$. By taking the relativistic and ponderomotive nonlinearity under consideration and hence, using the second order correction in the electron density equation (with the help of ponderomotive force), total density can be expressed as $[3,10]$ :

$$
n=n_{0}\left[1+\frac{c^{2}}{\omega_{p 0}^{2}}\left(\nabla^{2} \gamma-\frac{(\nabla \gamma)^{2}}{\gamma}\right)\right],
$$

where $n_{0}$ is the equilibrium electron density in absence of laser beam, $n$ is the modified density of plasma electrons due to relativistic and ponderomotive force. $\omega_{p 0}$ is the plasma frequency given by $\omega_{p 0}^{2}=\frac{4 \pi n_{0} e^{2}}{m_{0}}$ (where $e$ is the electron charge, $m_{0}$ its rest mass).

Following the standard techniques $[2-5,10]$, one obtains the equation governing the beam width parameter for laser beam (using normalization distance, $\left.\xi=z c / \omega_{0} r_{0}^{2}\right)$ as

$$
\begin{aligned}
& \frac{\mathrm{d}^{2} f_{0}}{\mathrm{~d} \xi^{2}}=\frac{1}{f_{0}^{3}}\left(-3 \alpha_{00}^{2}+8 \alpha_{02}+1-2 \alpha_{00}\right) \\
& -\frac{\omega_{p 0}^{2}}{\omega_{0}^{2}} \frac{e^{2} E_{00}^{2} k_{0}^{2} r_{0}^{2}}{2 \gamma^{3} m_{0}^{2} \omega_{0}^{2} c^{2} f_{0}^{2}}\left[\left(\alpha_{00}-1\right)\right. \\
& \left.+\frac{c^{2}}{\omega_{p 0}^{2} r_{0}^{2} f_{0}^{2}}\left(4 \alpha_{02}+\alpha_{00}\right)\right] .
\end{aligned}
$$

We obtain the following equation for $S_{02}, \alpha_{00}, \alpha_{02}$ as [4]:

$$
\begin{gathered}
\frac{\partial S_{02}}{\partial z}=-\frac{2 \alpha_{02}}{k_{0}^{2} r_{0}^{2} f_{0}^{6}}-\frac{3 \alpha_{02} \alpha_{00}}{2 k_{0}^{2} r_{0}^{2} f_{0}^{6}}-\frac{3 \alpha_{00}^{2}}{4 k_{0}^{2} r_{0}^{2} f_{0}^{6}} \\
+\frac{e E_{00}^{2} r_{0}^{2}}{m_{0}^{2} \omega_{0}^{2} c^{2} k_{0}^{2} \gamma^{3} f_{0}^{6}} \frac{-\alpha_{00}+2 \alpha_{02}}{r_{0}^{4} f_{0}^{4}} \\
+\frac{e E_{00}^{2}}{4 m_{0}^{2} \omega_{0}^{2} k_{0}^{2} c^{2} \gamma^{2}}\left(\frac{16 \alpha_{02}}{r_{0}^{2} f_{0}^{4}}-\frac{8 \alpha_{00}}{r_{0}^{2} f_{0}^{4}}\right) \\
\frac{\partial \alpha_{00}}{\partial z}=-\frac{16 S_{02} f_{0}^{2}}{r_{0}^{2}} \\
\text { and } \quad \frac{\partial \alpha_{02}}{\partial z}=\frac{8 S_{02} f_{0}^{2}}{r_{0}^{2}}-\frac{24 \alpha_{00} S_{02} f_{0}^{2}}{r_{0}^{2}}
\end{gathered}
$$

the beam width parameters $f_{0}$ of the laser beam depend on the coefficients $\left(\alpha_{00}\right.$ and $\left.\alpha_{02}\right)$ of $r^{2}$ and $r^{4}$ in extended-paraxial region. In order to have a numerical appreciation of contribution of off-axial rays in the beam width parameter (when both the relativistic and ponderomotive nonlinearity are taken into account), we have performed numerical computation of Eq. (2) with the help 
of Eqs. (4), (5) and (6). The coupled equations have been solved for an initial plane wave front; the initial conditions used here are $f_{0}=1$ and $\mathrm{d} f_{0} / \mathrm{d} z=0$ at $z=0$ and $S_{00}=S_{02}=0$ at $z=0$.

The results are presented in the form of Fig. 1. Figure 1a depicts the variation in laser beam intensity with normalized distance and radial distance, when the paraxial approximation is taken into consideration [by substituting $\alpha_{00}$ and $\alpha_{02}=0$ in Eq. (4)]. It is obvious from Fig. 1a that as the laser beam propagates through the plasma, it gets filamented. Figure 1a clearly expresses the generated filaments of the laser beam in the presence of both relativistic and ponderomotive nonlinearities. Figure $1 \mathrm{~b}$ shows the variation of the laser beam intensity with normalized distance and radial distance including the off-axial contribution. It can be clearly seen from Fig. 1b that the filaments are split when the off-axial part contributes to the beam and the point at which the laser focuses is also altered. It is obvious that in paraxial region the intensity of laser beams is maximum at $r=0$ along the distance of propagation as $\alpha_{00}=\alpha_{02}=0$, while in extended paraxial region the laser intensity becomes minimum at $r=0$, and maximum at $r= \pm 0.45$, as also was verified analytically by Eq. (2).

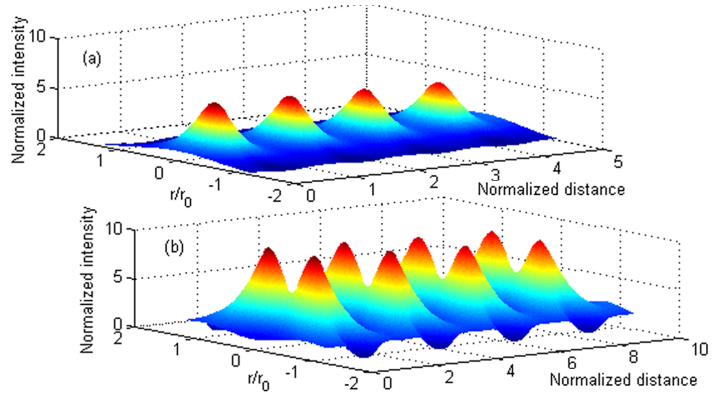

Fig. 1. Variation in laser beam width parameter with normalized distance when relativistic and ponderomotive nonlinearities are simultaneously operative, where red dashed line is for on axis (paraxial) case when $\alpha_{01}=\alpha_{02}=0$ and solid black line is for off-axial (extended paraxial) contribution when $\alpha_{01} \neq \alpha_{02} \neq 0$.

In Fig. 2 beam width parameter has been plotted for both the cases paraxial and modified paraxial. It can clearly be observed from Fig. 2 that focusing becomes faster in extended-paraxial case in comparison to paraxial case due to the participation of off-axis parts $\left(\alpha_{00} \neq\right.$ $\alpha_{02} \neq 0$ ). In Eq. (4) the results for paraxial approximation can be reproduced by substituting $\alpha_{00}=\alpha_{02}=0$. It is also observed (by solving Eq. (2), results are not presented here) that in paraxial case, the intensity of laser beam is maximal at $r=0$ along the distance of propagation as $\alpha_{00}=\alpha_{02}=0$. The following set of the laser beam parameters have been used in the numerical calculation: the vacuum wavelength of $\mu$; the laser beam $(\lambda=1064 \mathrm{~nm})$; the initial radius of the laser beams $(20 \mu \mathrm{m})$; laser power flux $\left(10^{18} \mathrm{~W} / \mathrm{cm}^{2}\right)$ at plasma density $\omega_{p}=0.06 \omega_{0}$; and $v_{\mathrm{th}}=0.1 c$.

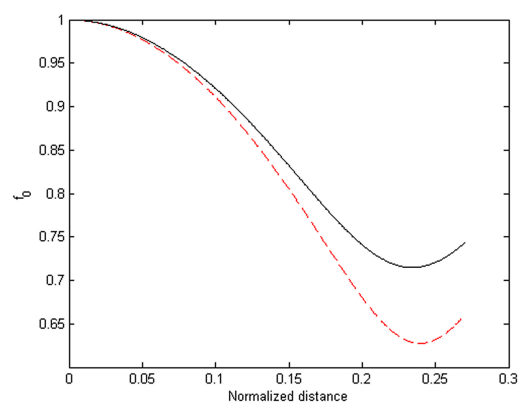

Fig. 2. Variation in normalized second harmonic power with normalized distance when relativistic and ponderomotive nonlinearities are simultaneously operative, red dashed line is for on axis (paraxial) when $\alpha_{0 h}=\alpha_{2 h}=0$ and black solid line is for off-axial (extended paraxial) contribution when $\alpha_{0 h} \neq \alpha_{2 h} \neq 0$.

On account of the change in the background density due to ponderomotive force and the relativistic effect, the laser beam gets filamented. Following standard procedure $[2-5,10]$, the equation governing the electron plasma wave generation can be written as

$$
\begin{gathered}
\frac{\partial^{2} N}{\partial t^{2}}-v_{\mathrm{th}}^{2} \nabla^{2} N+2 \Gamma_{e} \frac{\partial N}{\partial t}-\frac{e}{m} \nabla(N E) \\
=\nabla\left[\frac{N}{2} \nabla\left(V V^{*}\right)-V \frac{\partial N}{\partial t}\right],
\end{gathered}
$$

where $2 \Gamma_{e}$ is the Landau damping factor, $v_{\mathrm{th}}^{2}$ is the square of the electron thermal speed, $E$ is the sum of electric vectors of electromagnetic wave and the self-consistent field, $V$ is the sum of drift velocities of the electron in the electromagnetic field and self-consistent field and $m$ is the relativistic mass of electrons. The density component varying at the pump wave frequency $\left(\omega_{0}\right)$ can be written as

$$
\begin{aligned}
& -\omega_{0}^{2} N_{1}+2 \mathrm{i} \omega_{0} \Gamma_{e} N_{1}-v_{\mathrm{th}}^{2} \nabla^{2} N_{1}+\frac{\omega_{p 0}^{2}}{\gamma}\left(\frac{n}{n_{0}}\right) N_{1} \\
& =\frac{e}{m_{0} \gamma}\left[n \nabla E_{0}+E_{0} \nabla n\right],
\end{aligned}
$$

where $V_{0}$ is the oscillation velocity of the electron in the pump wave field and $n$ is the time independent component of electron density. It is obvious from the source term of Eq. (7) that one component of $N_{1}$ varies as $E_{0}$. Therefore $N_{1}$ can be written as

$$
N_{1}=N_{10}^{\prime}(r, z) \mathrm{e}^{-\mathrm{i} k z-\mathrm{i} k s}+N_{20}^{\prime}(r, z) \mathrm{e}^{-\mathrm{i} k_{0} z-\mathrm{i} k_{0} s_{0}},
$$

where $k^{2}=\left(\omega_{0}^{2}-\omega_{p 0}^{2}\right) / v_{\mathrm{th}}^{2}, \quad k_{0}=\left(\omega_{0} / c\right) \varepsilon_{f}^{1 / 2}$ and $N_{10}^{\prime}(x, y, z)$ and $N_{20}^{\prime}(x, y, z)$ are the complex functions of their arguments [6], and the profile of $N_{20}^{\prime}$ comes out as

$$
\begin{gathered}
N_{20}^{\prime} \approx-\left\{N _ { 0 e } e ^ { 3 } E _ { 0 0 } ^ { 3 } \operatorname { e x p } ( - \frac { r ^ { 2 } } { 2 r _ { 0 } ^ { 2 } f _ { 0 } ^ { 2 } } ) \left[\left(\frac{2 \alpha_{00} r}{r_{0}^{2} f_{0}^{2}}\right.\right.\right. \\
\left.+\frac{2 \alpha_{02} r^{3}}{r_{0}^{4} f_{0}^{4}}\right)-\frac{2 r}{r_{0}^{2} f_{0}^{2}}\left(1+\frac{\alpha_{00} r^{2}}{r_{0}^{2} f_{0}^{2}}+\frac{\alpha_{02} r^{4}}{r_{0}^{4} f_{0}^{4}}\right)
\end{gathered}
$$




$$
\begin{aligned}
& -\frac{f_{0}^{2} v_{\mathrm{th}}^{2}}{V_{00}^{2}}\left(\left(1+\frac{\alpha_{00} r^{2}}{r_{0}^{2} f_{0}^{2}}+\frac{\alpha_{02} r^{4}}{r_{0}^{4} f_{0}^{4}}\right)^{-1}\right) \\
& \left.\left.\times\left(\frac{\alpha_{00} r}{r_{0}^{2} f_{0}^{2}}+\frac{2 \alpha_{02} r^{3}}{r_{0}^{4} f_{0}^{4}}\right)-\frac{r}{r_{0}^{2} f_{0}^{2}}\right]\right\} \\
& /\left[4 v_{\mathrm{th}}^{2} m_{0}^{3} \gamma \omega_{0}^{2} f_{0}^{3}\right. \\
& \left.\times\left(\omega_{0}^{2}-k_{0}^{2} v_{\mathrm{th}}^{2}-2 \mathrm{i} \omega_{0} \Gamma_{e}-\omega_{p 0}^{2} n / \gamma n_{0}\right)\right] .
\end{aligned}
$$

\section{Second harmonic generation}

This plasma wave generated at the pump frequency can interact with the incident laser beam and a second harmonic can be generated. After obtaining the expression for $N_{20}^{\prime}$ by Eq. (9), we obtain the expression for the second-harmonic yield. The wave equation governing the second harmonic generation is given by

$$
\nabla^{2} A_{2}+\frac{\omega_{2}^{2}}{c^{2}} \varepsilon_{2}\left(\omega_{2}\right) A_{2}=\frac{\omega_{p 0}^{2}}{c^{2}} \frac{N_{1}}{n_{0}} A_{0},
$$

where $\omega_{2}=2 \omega_{0}$ and $\varepsilon_{2}\left(\omega_{2}\right)$ are the effective dielectric constants of the plasma at the fundamental and second harmonic frequency.

The solution of Eq. (10) in terms of vector potential can be written as

$$
A_{2}=A_{20}^{\prime}(x, y, z) \mathrm{e}^{-\mathrm{i} k_{2} z}+A_{21}^{\prime}(x, y, z) \mathrm{e}^{-2 \mathrm{i} k_{0} z} .
$$

We have taken only that component of $N_{1}$ in Eq. (10) which arises on account of the source term of Eq. (8), because it is not Landau damped. Furthermore one can write

$$
\begin{aligned}
& A_{20}^{\prime}=A_{20}(x, y, z) \mathrm{e}^{-\mathrm{i} S_{2}} \\
& \text { and } A_{21}^{\prime}=A_{21}(x, y, z) \mathrm{e}^{-2 \mathrm{i} S_{0}} .
\end{aligned}
$$

To solve Eq. (10), let the solution be

$$
\begin{aligned}
& A_{20}^{2}=\frac{B^{\prime}}{f_{2}^{2}}\left(1+\frac{\alpha_{0 h} r^{2}}{b_{0}^{2} f_{2}^{2}}+\frac{\alpha_{2 h} r^{4}}{b_{0}^{4} f_{2}^{4}}\right) \exp \left(-\frac{r^{2}}{b_{0}^{2} f_{2}^{2}}\right), \\
& S_{2}=\frac{S_{0 h}}{b_{0}^{2}}+\frac{S_{2 h}}{b_{0}^{4}} \quad \text { with } \quad S_{0 h}=\frac{r^{2}}{2 f_{2}} \frac{\mathrm{d} f_{2}}{\mathrm{~d} z},
\end{aligned}
$$

and using (12) we get

$$
\begin{aligned}
A_{21} & =\frac{\omega_{p 0}^{2}}{c^{2}} \frac{\omega_{2}}{\omega_{0}}\left(\frac{N_{20}^{\prime}}{n_{0}}\right) \frac{E_{00}}{f_{0}}\left(1+\frac{\alpha_{0 h} r^{4}}{b_{0}^{2} f_{2}^{2}}+\frac{\alpha_{2 h} r^{4}}{b_{0}^{4} f_{2}^{4}}\right) \\
& \times \frac{\exp \left(-r^{2} / b_{0}^{2} f_{2}^{2}\right)}{k_{2}^{2}-4 k_{0}^{2}} .
\end{aligned}
$$

Here $b_{0}$ is the second harmonic beam width. The coefficients $\alpha_{0 h}$, etc. functions are the same as $\alpha_{00}$ etc. functions in the previous section. Further we use the initial conditions $\mathrm{d} f_{2} / \mathrm{d} z=0, f_{2}=1$ at $z=0$, and $S_{0 h}=S_{2 h}=0$ at $z=0, f_{2}$ is the dimensionless width parameter of the second harmonic radiation, given by

$$
\frac{\mathrm{d}^{2} f_{2}}{\mathrm{~d} \xi^{2}}=\frac{r_{0}^{4}}{b_{0}^{4}} \frac{k_{0}^{2}}{k_{2}^{2}} \frac{1}{f_{2}^{3}}\left(-3 \alpha_{0 h}^{2}+8 \alpha_{2 h}+1+2 \alpha_{0 h}\right)
$$

$$
\begin{aligned}
& -f_{2} \frac{c^{2}}{v_{\mathrm{th}}^{2}}\left(\frac{k_{0}}{k_{2}}\right)^{2} \frac{\omega_{p 0}^{2}}{\omega_{2}^{2}} \frac{e^{2} E_{00}^{2} k_{0}^{2} r_{0}^{2}}{2 m_{0}^{2} \omega_{0}^{2} c^{2} f_{0}^{2}} \\
& \times\left[\left(1-\alpha_{00}\right)+\frac{c^{2}}{\omega_{p 0}^{2} r_{0}^{2} f_{0}^{2}}\left(4 \alpha_{02}+\alpha_{00}\right)\right] .
\end{aligned}
$$

We obtain the following equation for $S_{2 h}, \alpha_{0 h}, \alpha_{2 h}$ as [4]:

$$
\begin{gathered}
\frac{\partial S_{2 h}}{\partial z}=\frac{-10 \alpha_{0 h} \alpha_{2 h}+2 \alpha_{0 h}^{3}+2 \alpha_{0 h}^{2}-4 \alpha_{2 h}}{2 k_{2}^{2} b_{0}^{2} f_{2}^{6}} \\
+\frac{\omega_{p 0}^{2}}{\omega_{2}^{2}} \frac{e^{2} E_{00}^{2} r_{0}^{2}}{2 \gamma^{3} m_{0}^{2} \omega_{0}^{2} c^{2} f_{0}^{2}}\left(\frac{2 \alpha_{02}}{r_{0}^{4} f_{0}^{4}}-\frac{\alpha_{00}}{r_{0}^{4} f_{0}^{4}}\right) \\
+\frac{e E_{00}^{2}}{m_{0}^{2} \omega_{2}^{2} c^{2} k_{2}^{2} \gamma^{2}}\left(\frac{4 \alpha_{02}}{r_{0}^{2} f_{0}^{4}}-\frac{2 \alpha_{00}}{r_{0}^{2} f_{0}^{4}}\right), \\
\frac{\partial \alpha_{0 h}}{\partial z}=-\frac{16 S_{2 h} f_{2}^{2}}{b_{0}^{2}}, \\
\frac{\partial \alpha_{2 h}}{\partial z}=\frac{8 S_{2 p} f_{2}^{2}}{b_{0}^{2}}-\frac{24 \alpha_{0 h} S_{2 h} f_{2}^{2}}{b_{0}^{2}} .
\end{gathered}
$$

The constants $B^{\prime}$ and $b_{0}$ are also determined by the boundary condition that the second harmonic generation is zero at $z=0$ :

$$
\begin{aligned}
B^{\prime} & \approx-\left\{N _ { 0 e } e ^ { 3 } E _ { 0 0 } ^ { 3 } \mathrm { e } ^ { - r ^ { 2 } / 2 r _ { 0 } ^ { 2 } } \left[\left(\frac{2 \alpha_{00} r}{r_{0}^{2}}+\frac{2 \alpha_{02} r^{3}}{r_{0}^{4}}\right)-\frac{2 r}{r_{0}^{2}}\right.\right. \\
\times(1+ & \left.\frac{\alpha_{00} r^{2}}{r_{0}^{2} f_{0}^{2}}+\frac{\alpha_{02} r^{4}}{r_{0}^{4} f_{0}^{4}}\right)-\frac{v_{\mathrm{th}}^{2}}{V_{00}^{2}}\left(1+\frac{\alpha_{00} r^{2}}{r_{0}^{2} f_{0}^{2}}+\frac{\alpha_{02} r^{4}}{r_{0}^{4} f_{0}^{4}}\right)^{-1} \\
& \left.\left.\times\left(\frac{\alpha_{00} r}{r_{0}^{2}}+\frac{2 \alpha_{02} r^{3}}{r_{0}^{4}}\right)-\frac{r}{r_{0}^{2}}\right]\right\} /\left[4 v_{\mathrm{th}}^{2} m_{0}^{3} \gamma \omega_{0}^{2}\right. \\
& \times\left(\omega_{0}^{2}-k_{0}^{2} v_{\mathrm{th}}^{2}-2 \mathrm{i} \omega_{0} \Gamma_{e}-\omega_{p 0}^{2} n / \gamma m_{0}\right) \\
& \left.\times\left(1+\frac{\alpha_{0 h} r^{2}}{b_{0}^{2}}+\frac{\alpha_{2 h} r^{4}}{b_{0}^{4}}\right)^{1 / 2} \mathrm{e}^{-r^{2} / 2 b_{0}^{2}}\left(k_{2}^{2}-4 k_{0}^{2}\right)\right]
\end{aligned}
$$

and $b_{0}=r_{0}$.

here,

$$
\begin{aligned}
A_{2} & =-E_{00} \frac{\omega_{p 0}^{2}}{c^{2}}\left[\frac{H_{1}}{f_{2}} \mathrm{e}^{-\left(\frac{r^{2}}{2 b_{0}^{2} f_{2}^{2}}+\frac{r^{2}}{2 r_{0}^{2}}\right)} \mathrm{e}^{-\mathrm{i}\left(S_{2}+k_{2} z\right)}\right. \\
& \left.-\frac{H_{2}}{f_{0}^{3}} \mathrm{e}^{-\left(\frac{r^{2}}{2 b_{0}^{2} f_{2}^{2}}+\frac{r^{2}}{r_{0}^{2} f_{0}^{2}}\right)} \mathrm{e}^{-2 \mathrm{i}\left(k_{0} z+S_{0}\right)}\right]
\end{aligned}
$$$$
\text { Using Eqs. (13), (14), (15a-c) and (16), we get }
$$$$
H_{1}=-\left\{e ^ { 3 } E _ { 0 0 } ^ { 3 } ( 1 + \frac { \alpha _ { 0 0 } r ^ { 2 } } { r _ { 0 } ^ { 2 } } + \frac { \alpha _ { 0 2 } r ^ { 4 } } { r _ { 0 } ^ { 4 } } ) \left[\left(\frac{2 \alpha_{00} r}{r_{0}^{2}}\right.\right.\right.
$$$$
\left.+\frac{2 \alpha_{02} r^{3}}{r_{0}^{4}}\right)-\frac{2 r}{r_{0}^{2}}\left(1+\frac{\alpha_{00} r^{2}}{r_{0}^{2} f_{0}^{2}}+\frac{\alpha_{02} r^{4}}{r_{0}^{4} f_{0}^{4}}\right)-\frac{v_{\mathrm{th}}^{2}}{V_{00}^{2}}
$$ 


$$
\begin{aligned}
\times(1+ & \left.\left.\left.\frac{\alpha_{00} r^{2}}{r_{0}^{2} f_{0}^{2}}+\frac{\alpha_{02} r^{4}}{r_{0}^{4} f_{0}^{4}}\right)^{-1}\left(\frac{\alpha_{00} r}{r_{0}^{2}}+\frac{2 \alpha_{02} r^{3}}{r_{0}^{4}}\right)-\frac{r}{r_{0}^{2}}\right]\right\} \\
& /\left[4 v _ { \mathrm { th } } ^ { 2 } m _ { 0 } ^ { 3 } \gamma _ { r = 0 } \omega _ { 0 } ^ { 2 } \left(\omega_{0}^{2}-k_{0}^{2} v_{\mathrm{th}}^{2}-2 \mathrm{i} \omega_{0} \Gamma_{e}\right.\right. \\
& \left.-\omega_{p 0}^{2} n / \gamma n_{0}\right)\left(1+\frac{\alpha_{0 h} r^{2}}{b_{0}^{2}}+\frac{\alpha_{2 h} r^{4}}{b_{0}^{4}}\right)^{1 / 2} \\
& \left.\times \exp \left(-\frac{r^{2}}{2 b_{0}^{2}}\right)\left(k_{2}^{2}-4 k_{0}^{2}\right)\right]
\end{aligned}
$$

and

$$
\begin{gathered}
H_{2}=-\frac{\omega_{2}}{\omega_{0}}\left\{e ^ { 3 } E _ { 0 0 } ^ { 3 } ( 1 + \frac { \alpha _ { 0 0 } r ^ { 2 } } { r _ { 0 } ^ { 2 } } + \frac { \alpha _ { 0 2 } r ^ { 4 } } { r _ { 0 } ^ { 4 } } ) \left[\left(\frac{2 \alpha_{00} r}{r_{0}^{2} f_{0}^{2}}\right.\right.\right. \\
\left.+\frac{2 \alpha_{02} r^{3}}{r_{0}^{4} f_{0}^{4}}\right)-\frac{2 r}{r_{0}^{2} f_{0}^{2}}\left(1+\frac{\alpha_{00} r^{2}}{r_{0}^{2} f_{0}^{2}}+\frac{\alpha_{02} r^{4}}{r_{0}^{4} f_{0}^{4}}\right)-\frac{f_{0}^{2} v_{\mathrm{th}}^{2}}{V_{00}^{2}} \\
\left.\left.\times\left(1+\frac{\alpha_{00} r^{2}}{r_{0}^{2} f_{0}^{2}}+\frac{\alpha_{02} r^{4}}{r_{0}^{4} f_{0}^{4}}\right)^{-1}\left(\frac{\alpha_{00} r}{r_{0}^{2} f_{0}^{2}}+\frac{2 \alpha_{02} r^{3}}{r_{0}^{4} f_{0}^{4}}\right)-\frac{r}{r_{0}^{2} f_{0}^{2}}\right]\right\} \\
/\left[4 v _ { \mathrm { th } } ^ { 2 } m _ { 0 } ^ { 3 } \gamma \omega _ { 0 } ^ { 2 } f _ { 0 } \left(\omega_{0}^{2}-k_{0}^{2} v_{\mathrm{th}}^{2}-2 \mathrm{i} \omega_{0} \Gamma_{e}\right.\right. \\
\left.\left.-\omega_{p 0}^{2} n / \gamma n_{0}\right)\left(k_{2}^{2}-4 k_{0}^{2}\right)\right]
\end{gathered}
$$

The power of the second harmonic wave $\left(P_{2}\right)$ incident across the transverse cross-section at $z$ is given by

$$
\begin{aligned}
P_{2} & =\frac{\omega_{o}^{2}}{8 \pi c} \int_{-\infty}^{\infty} \mathrm{d} x \int_{-\infty}^{\infty} A_{2} A_{2}^{*} \mathrm{~d} y=P_{0}\left(\frac{\omega_{p 0}^{2} r_{0}}{c^{2}}\right)^{2} \\
\times & {\left[\frac{H_{2}^{2} f_{0}^{2}\left(1+\frac{\alpha_{00} r^{2}}{r_{0}^{2} f_{0}^{2}}+\frac{\alpha_{02} r^{4}}{r_{0}^{4} f_{0}^{4}}\right)^{1 / 2}}{9\left(1+\frac{\alpha_{0 h} r^{2}}{b_{0}^{2}}+\frac{\alpha_{2 h} r^{4}}{b_{0}^{4}}\right)^{1 / 2}}\right.} \\
- & \frac{\left(1+f_{2}^{2}\right)^{2}\left(1+\frac{\alpha_{0 h} r^{2}}{b_{0}^{2}}+\frac{\alpha_{2 h} r^{4}}{b_{0}^{4}}\right)^{4}\left(1+\frac{\alpha_{00} r^{2}}{r_{0}^{2} f_{0}^{2}}+\frac{\alpha_{02} r^{4}}{r_{0}^{4} f_{0}^{4}}\right)^{1 / 2}}{8 H_{2} f_{0}^{4} f_{2}^{4}\left(1+\frac{\alpha_{00} r^{2}}{r_{0}^{2} f_{0}^{2}}+\frac{\alpha_{02} r^{4}}{r_{0}^{4} f_{0}^{4}}\right)^{1 / 2}} \\
- & \frac{\left(f_{0}^{3}+3 f_{2}^{2}+f_{0}^{2} f_{2}^{2}\right)^{2}\left(1+\frac{\alpha_{0 h} r^{2}}{b_{0}^{2}}+\frac{\alpha_{2 h} r^{4}}{b_{0}^{4}}\right)^{1 / 2}}{\left.\cos \left(\left(k_{2}-2 k_{0}\right) 2 z\right)\right],}
\end{aligned}
$$

where $P_{0}=\left(\omega_{0}^{2} / 8 \pi c\right) \pi r_{0}^{2} A_{00}^{2}$. In Sect. 3 , the phenomenon of harmonic generation is surveyed in the presence of split filamentary structures of an ultra intense laser pulse. The expression for the beam width parameter and power of the second harmonic has been derived in the presence of both relativistic and ponderomotive nonlinearities. In order to observe the second harmonic yield $P_{2} / P_{0}$ with the distance of propagation, we have solved Eq. (18) numerically and the results are presented in the form of Fig. 3. Figure 3 has been plotted with and

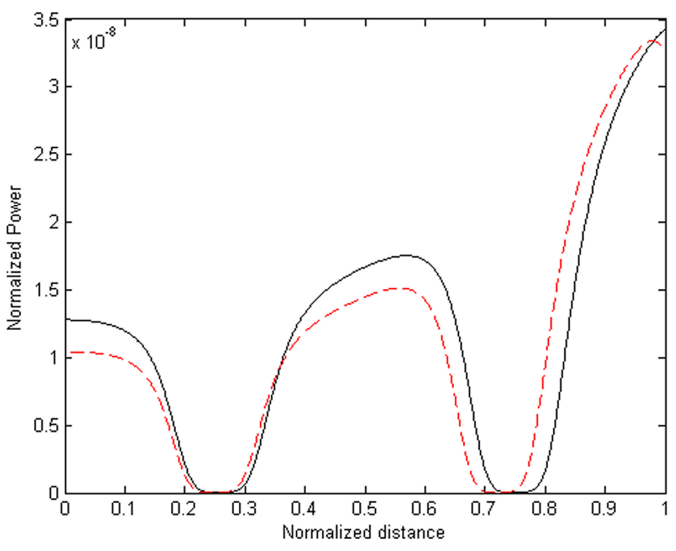

Fig. 3. Variation in normalized power of second harmonic with normalized distance $(\xi)$. when both relativistic and ponderomotive nonlinearities are operative. Red dashed line is for axial and solid black line is for off-axial contribution.

without the contribution of the coefficients $\alpha_{00}$ and $\alpha_{02}$ and explicitly illustrates the comparison of the second-harmonic yield $P_{2} / P_{0}$ in the off-axis $\left(\alpha_{00} \neq \alpha_{02} \neq 0\right)$ and on-axis cases $\left(\alpha_{00}=\alpha_{02}=0\right)$ of the laser beam. Figure 3 shows the variation of the power of the second harmonic with normalized distance when the paraxial as well as extended paraxial approximation has been used. It clearly expresses the contribution of $r^{2}$ and $r^{4}$ coefficients on the power of second harmonic in the presence of ponderomotive and relativistic nonlinearity, the solid black line shows when restriction on the laser beam is relaxed while red dashed line shows the same with restricted laser beam profile. The second-harmonic yield is about an order of magnitude higher when the off-axis case is taken. It is also obvious from this figure that the second-harmonic yield increases in the off-axis case. This is due to the fact that, by increasing the distance in off-axis case, the self-focusing of the laser beam becomes faster with steep transverse intensity gradients; therefore, the plasma wave generation and, hence, second-harmonic yield also increases accordingly.

\section{Conclusion}

In this work, it is observed that the filamentation process becomes faster in comparison to the case when the off-axial contribution is not taken into account. Due to the contribution of the off-axial part the filamentary structures of the laser beam get modified, these modified structures affect the plasma wave generation. Second-harmonic yield has also been studied by considering extended paraxial contribution and it is observed that in 
presence of off-axis part second-harmonic yield is higher in magnitude. So one can infer that the presence of off-axial rays contributes significantly to the phenomenon of harmonic generation and hence harmonic yield. The second-harmonic yield can be used as a diagnostic tool for studying the relativistic and ponderomotive filamentation process in the laboratories.

\section{Acknowledgments}

This work was partially supported by the Madhya Pradesh Council for Science and Technology, Bhopal, India.

\section{References}

[1] M.S. Sodha, A. Sharma, Phys. Plasmas 14, 044501 (2007).

[2] S.A. Akhmanov, A.P. Sukhorukov, K.V. Khokhlov, Sov. Phys. Uspekhi 10, 609 (1968); also Usp. Fiz. Nauk 93, 19 (1967).
[3] P. Sharma, S.T. Mahmud, M.K. Gupta, R.P. Sharma, IEEE Trans. Plasma Sci. 37, 2158 (2009).

[4] R. Gupta, P. Sharma, P.K. Chauhan, M. Rafat, R.P. Sharma, Phys. Plasma 16, 043101 (2009).

[5] R.P. Sharma, P. Sharma, P.K. Chauhan, Phys. Plasma 14, 103112 (2007).

[6] P. Sharma, S.T. Mahmood, M.K. Gupta, R.P. Sharma, Phys. Plasma 15, 042105 (2008).

[7] A. Pukhov, Z.-M. Sheng, J. Meyer-ter-Vehn, Phys. Plasma 6, 2847 (1999).

[8] J.A. Stamper, R.H. Lehmberg, A. Schmitt, M.J. Herbst, F.C. Young, J.H. Gardner, S.P. Obenschain, Phys. Fluids 28, 2563 (1985).

[9] J. Meyer, Y. Zhu, Phys. Fluids 30, 890 (1987).

[10] M.S. Sodha, A.K. Ghatak, V.K. Tripathi, Prog. Opt. 13, 171 (1976a). 